\title{
Endoscopic transnasal dacryocystorhinostomy
}

\author{
M. McDonogh and J. H. MeIrInG* (Pretoria, R.S.A.)
}

\begin{abstract}
The rigid Hopkins endoscope has been applied to simplify the operation of dacryocystorhinostomy, preventing unnecessary trauma to the medial orbital tissues. The success of the surgical technique is absolutely dependent on a thorough knowledge of the relevant surgical anatomy.
\end{abstract}

\section{Introduction}

During a routine functional endoscopic sinus operation the nasolacrimal duct was inadvertently exposed. This started a train of thought to apply it to the advantage of patients with lacrimal duct obstruction. The method described below is a direct transnasal approach using a rigid Storz Hopkins rodlens endoscope with a $30^{\circ}$ viewing angle. This brings the field of vision right into the nose, directly onto the operated area. The operation is therefore easier and more exact with less tissue trauma than the external approach next to the eye.

\section{Surgical anatomy}

The anatomical relationships of the lacrimal sac and nasolacrimal duct as they relate to the lateral nasal wall was investigated on dry skulls, anatomical dissection material, fresh cadavers, X-ray plates, CT scans and anatomy textbooks. Before reading any further it might be advisable to imagine the positioning of these structures in relation to the lateral nasal wall.

The lacrimal sac $(12-15 \mathrm{~mm}$. long) is housed in the lacrimal fossa between the anterior and posterior lacrimal crests. The anterior lacrimal crest is formed by the frontal process of the maxillary bone and the posterior crest by the lacrimal bone. The nasolacrimal duct (18 mm. long) leads from the sac into the lateral wall of the nose. The bony canal for the duct is formed by the maxilla, lacrimal bone and inferior nasal concha (Fig. $1 \mathrm{a})$.

The nasal surface anatomy of these structures is as follows: The nasal vestibule leads into the nasal atrium which lies between the ethmoidal and the conchal crests of the maxilla. This atrium is concave anteriorly but has a vertical convex ridge just before the atrium leads into the middle meatus (Figs. 1b, 3). This ridge extends from the highest point of the inferior turbinate upwards and ends immediately in front of the middle turbinate's attachment. This ridge corresponds to the frontal process of the maxilla immediately anterior to its junction with the lacrimal bone. The lacrimal bone is paper thin making identification of this junction with the hard frontal process very easy, vide infra. This vertical junction lies approximately $3 \mathrm{~mm}$. behind the ridge and extends upwards under the anterior attachment of the middle turbinate and above the ethmoid crest for approximately $5 \mathrm{~mm}$. (Fig. 1b). The nasolacrimal duct and sac lie immediately lateral to this junction in a vertical plane, i.e. just behind this ridge and just in front of the middle turbinate's anterior edge (Fig. 3). The duct disappears under the inferior turbinate to open into the inferior meatus (Fig. 2).

Superiorly, the duct joins the sac halfway between the attachments of the middle and inferior turbinates. The superior border of the lacrimal sac is above the middle turbinate's anterior attachment and is lateral to the agger nasi cells. Posterior to the ridge there is a slight concave depression immediately in front of the uncinate process at the entrance to the middle meatus. The uncinate process marks the entrance to the ethmoid labyrinth. This process is attached to the lacrimal bone and

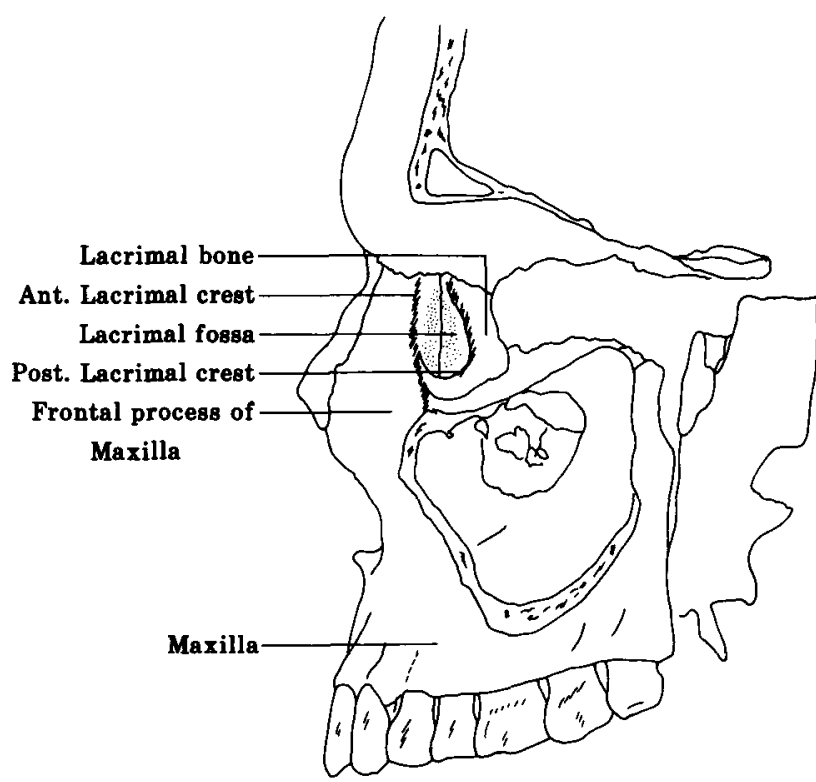

Fig. 1a

Medial wall or orbital cavity. 


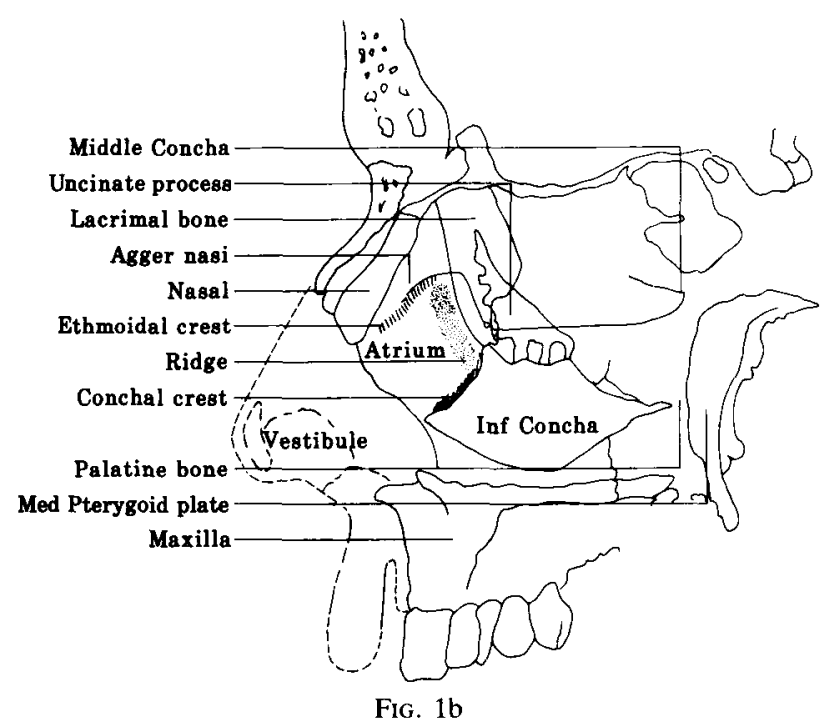

Lateral wall of nasal cavity.

the inferior turbinate by a series of delicate footpieces (Fig. 1b). The anterior footpieces are at the junction of the lacrimal fossa and the orbital plate of the lacrimal bone. This is an important point to remember because entry beyond this point in a lateral direction takes you behind the orbital septum and into orbital fat. The anterior footpieces of the uncinate process should therefore be regarded as the extreme posterior limit of safety.

\section{Surgical technique}

The instruments used are a $30^{\circ}$ Storz Hopkins rodlens endoscope, a Rosen curette, a gently curved suction cannula, a blunt ended $90^{\circ}$ probe and a fine back-biting guillotine. A fine forward biting Hyjack forceps could also be used.

The procedure can be done under local or general anaesthesia. Pack the anterior nasal cavity with cocaine impregnated gauze after infiltrating the ridge area with 1:80,000 adrenaline and lignocaine. This gives easy access, mucosal anaesthesia and a bloodless field. Flatten the infiltrated mucosal area down with a Freer dissector. Use the Rosen curette to make a vertical incision in the lateral nasal wall mucosa just in front of the ridge extending from above the middle turbinate to the top of the inferior turbinate. Dissect the mucous membrane off the bone in a posterior direction until you reach the base of the uncinate process. Remove the mobilised mucosa. Now palpate the exposed bone just behind the ridge from anterior to posterior with the Rosen. Use firm lateral pressure (Fig. 4).

The lacrimal bone, being papery thin, gives way as soon as you reach the junction. The duct lies immediately lateral to this point. Slide the curette upwards under the middle turbinate's attachment and downwards, level with the lower edge of the middle turbinate. Now insert the guillotine into the vertical groove thus formed, and remove the maxillary part of the fossa anteriorly going as high up under the middle turbinate as possible and about half-way down the length of the duct. Then remove the lacrimal part of the fossa to the base of the uncinate process, being very careful posterolaterally (Fig. 4). Finger pressure on the sac area from outside bulges it into the nasal cavity. Cut an inferiorly based flap in the medial wall of the sac, fold it down and suture it onto the inferior turbinate (Fig. 4). The lacrimal cannaliculi can now be probed at this stage if necessary.

Tidy up the bony and mucosal edges, insert a silastic "shoe-horn" into the remaining sac and duct if necessary and place a melolite dressing (Smith and Nephew) into the atrium and middle meatus. Remove the dressing 8-24 hours later and the silastic two weeks later.

\section{Discussion}

This operation is a conservative and direct one and is easily learned by the ear nose and throat specialist and opthalmologist alike. It is far less traumatic than the external approach as there is no facial scar, no disruption of the medial palpebral ligaments or of the angular facial vessels. The lacrimal apparatus is often involved with facial fractures. These are difficult cases as there are multiple problems involving the orbit, nose and eth-

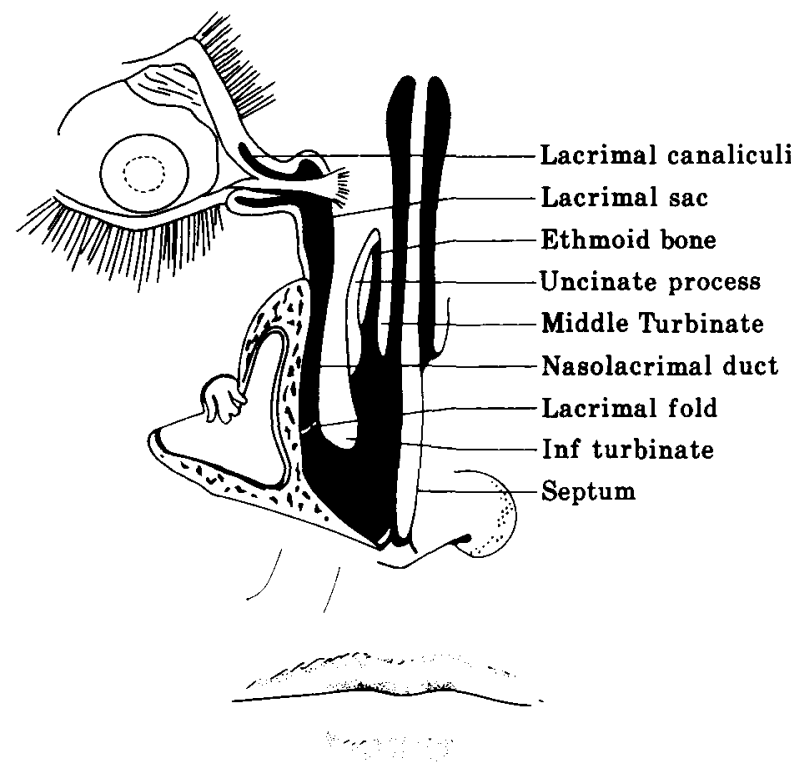

Fig. 2

Coronal section through lacrimal sac and duct.

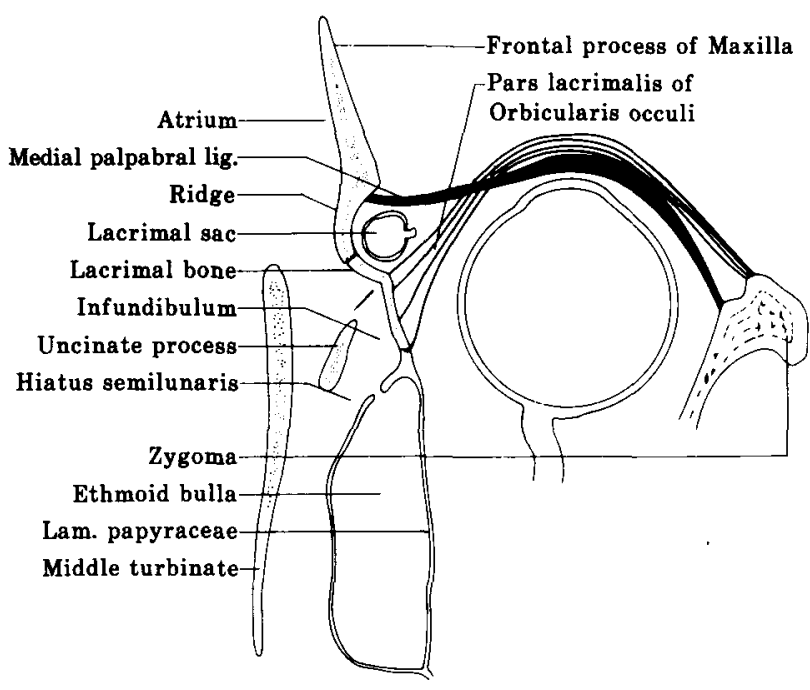

FlG. 3

Transverse section through lacrimal sac. 

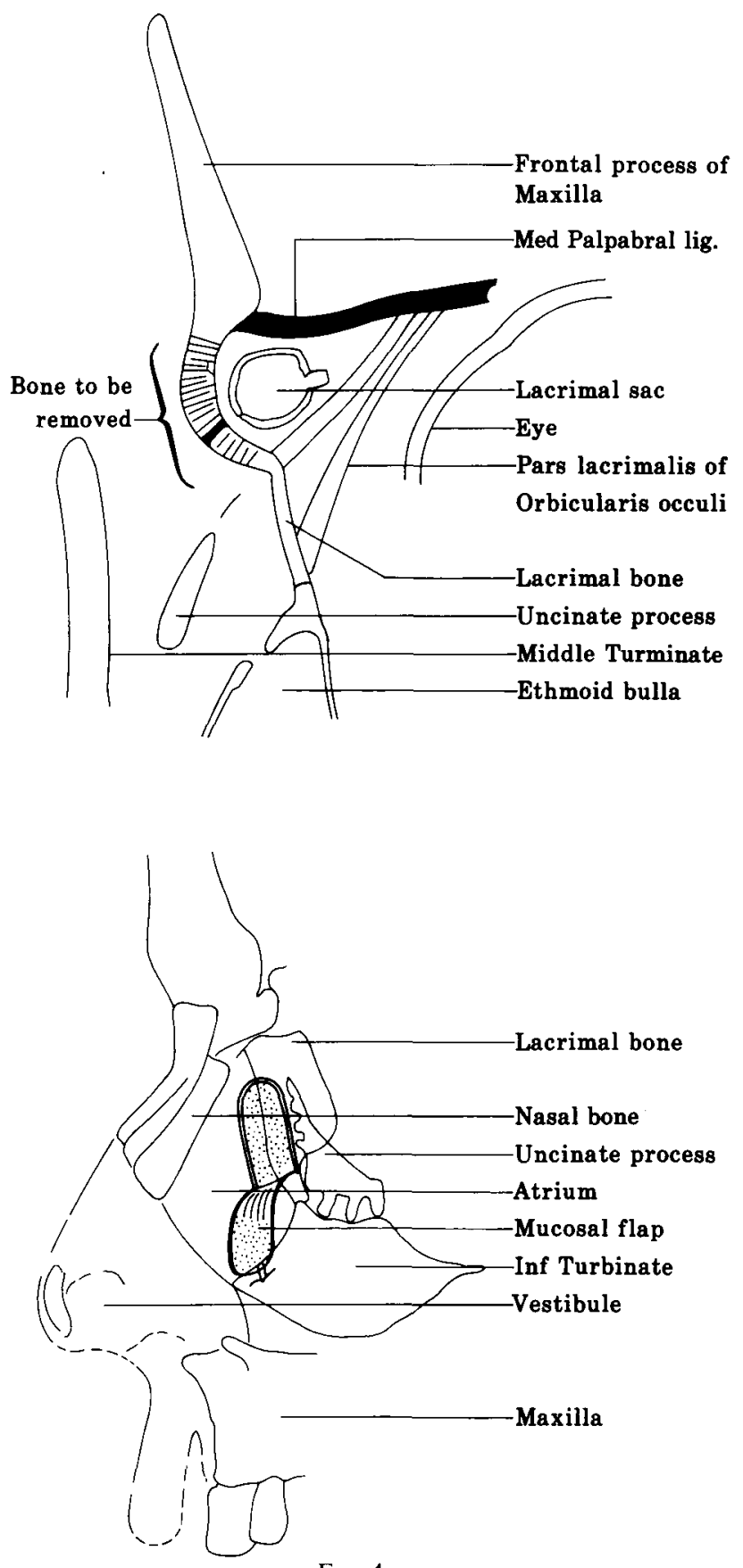

FIG. 4

Bone removal and flap. moids, often with avulsion of the sac, cannaliculi or duct or crushing of the tissues leading to malfunction.

Locating the sac can be a problem in these cases. A probe can be passed via a cannaliculus medially and gently forced between fracture fragments to present in the nose. Shining a cold light from the outside over the lacrimal fossa can also help identify the nasal relationship of the sac. Care should be taken not to press the light against the skin as it will cause a skin burn.

Difficult or revision cases should initially be approached endoscopically but if difficulties arise don't be afraid to resort to the classic external approach as this remains a good operation for the right indications. It is urged that surgeons should gain appropriate experience in functional nasoendoscopic surgery before accepting patients for this procedure.

\section{Acknowledgements}

We would like to thank Steve Kloppers for introducing the author to the nasal endoscope as well as Stuart Hofmeyr and Gustav Joyce for their continual support and encouragement.

Address for correspondence:

Dr. M. McDonogh,

Medforum 409

412 Schoeman St.,

Pretoria 0002, R.S.A. 